\title{
Magnetic shuffling of coronal downdrafts ${ }^{\star}$
}

\author{
A. Petralia ${ }^{1,2}$, F. Reale ${ }^{1,2}$, and S. Orlando ${ }^{2}$ \\ ${ }^{1}$ Dipartimento di Fisica \& Chimica, Università di Palermo, Piazza del Parlamento 1, 90134 Palermo, Italy \\ e-mail: apetralia@astropa.unipa.it \\ 2 INAF-Osservatorio Astronomico di Palermo, Piazza del Parlamento 1, 90134 Palermo, Italy
}

Received 21 November 2016 / Accepted 19 January 2017

\begin{abstract}
Context. Channelled fragmented downflows are ubiquitous in magnetized atmospheres, and have recently been addressed based on an observation after a solar eruption.

Aims. We study the possible back-effect of the magnetic field on the propagation of confined flows.

Methods. We compared two 3D magnetohydrodynamic simulations of dense supersonic plasma blobs that fall down along a coronal magnetic flux tube. In one, the blobs move strictly along the field lines; in the other, the initial velocity of the blobs is not perfectly aligned with the magnetic field and the field is weaker.

Results. The aligned blobs remain compact while flowing along the tube, with the generated shocks. The misaligned blobs are disrupted and merge through the chaotic shuffling of the field lines. They are structured into thinner filaments. Alfvén wave fronts are generated together with shocks ahead of the dense moving front.

Conclusions. Downflowing plasma fragments can be chaotically and efficiently mixed if their motion is misaligned with field lines, with broad implications for disk accretion in protostars, coronal eruptions, and rain, for example.
\end{abstract}

Key words. magnetohydrodynamics (MHD) - Sun: corona - Sun: activity - accretion, accretion disks

\section{Introduction}

The corona is the outer part of the solar atmosphere. It is highly structured by the magnetic field, but it is also highly dynamic: the flows are generated by various mechanisms. For instance, compressions and rarefactions at the footpoints can trigger up- or downflows inside the magnetic channels (e.g., spicules, siphon flows). Depending on the speed, the upflowing plasma can be ejected outside the solar atmosphere (Chen 2011; Webb \& Howard 2012) and/or it falls back onto the surface (e.g., Innes et al. 2012, and references therein). Downfalling fragments after an eruption were used as a template for the accretion in young stars in both the high- $\beta$ and low- $\beta$ regimes (Reale et al. 2013, 2014; Petralia et al. 2016). The accreting cold material from the circumstellar disk flows along magnetic channels and impacts the stellar surface (Uchida \& Shibata 1984; Bertout et al. 1988). The structure and dynamics of the falling material is presumably influenced by both the strength and complexity of the magnetic fields and by the flow inhomogeneity (Matsakos et al. 2013; Orlando et al. 2013; Colombo et al. 2016). Downfalls can be also generated by thermal instability (Parker 1953; Field 1965) in the so-called coronal rain. In this case, a strong heating at loop footpoints can lead to a high plasma density in loops. The high radiative losses exceed the heating and cause a catastrophic plasma cooling and condensation (Antolin \& Rouppe van der Voort 2012; Kleint et al. 2014; Fang et al. 2015).

When the plasma falls in a region where the magnetic field is strong, it can be channelled along flux tubes. After a spectacular

\footnotetext{
* Movies associated to Figs. 2 and 3 are available at http://www . aanda.org
}

solar eruption on June 7, 2011, large fragments were spread all over the solar surface (van Driel-Gesztelyi 2014; Innes et al. 2012; Reale et al. 2013, 2014), and Petralia et al. (2016) studied some fragments that fell close to active regions and interacted strongly with the magnetic field. Magnetohydrodynamic (MHD) modelling showed that the shocks ahead of the downfalling fragments brighten the final segment of the magnetic channel. The model also showed that the plasma blobs are warped and further fragmented as soon as their interaction with the field becomes significant. It is clear that the plasma is conditioned by the field and the field by the plasma.

In the present work we investigate this interaction and how it can or cannot determine a significant disruption of the blobs. To this purpose, we compare two similar MHD simulations, one with, the other without this effect.

\section{MHD modelling}

As in Petralia et al. (2016), we study the propagation of plasma blobs inside a magnetized corona through detailed MHD modelling. Our model solves the same MHD equations as described in Petralia et al. (2016), including thermal conduction and radiative losses. The calculations are performed using the same MHD module available in PLUTO (Mignone et al. 2007, 2012), a modular, Godunov-type code for astrophysical plasmas. We use radiative losses from the CHIANTI code (Version 7; Landi et al. 2012), assuming a density of $10^{9} \mathrm{~cm}^{-3}$ and ionization equilibrium according to Dere (2009). We assume no losses and heating in the chromosphere and inside the initial cold blobs (i.e., for $\left.T \leq 10^{4} \mathrm{~K}\right)$. 
Our two simulations describe the evolution of four blobs moving across a magnetized coronal atmosphere. We compare a case in which the blobs are not fully channelled by the magnetic field to another in which they are. We consider a typical coronal field configuration with closed arch-like lines anchored in the photosphere (Reale 2014). This configuration has no special symmetry, and a full 3D description is necessary. However, we can consider a symmetric magnetic field with respect to a plane perpendicular to the surface. Regardless of the initial direction of the blobs, the field geometry and strength will prevent them from moving much across the field lines; therefore, the domain is not needed to be large in that direction, assumed to be the $Y$ direction. We approach the configuration of a loop-populated active region, still keeping it manageable and simple, with a combination of magnetic dipoles, such that the magnetic field is symmetric with respect to the side boundaries and is closed down in the chromosphere. The computational box is three-dimensional and Cartesian $X, Y, Z$, and $4 \times 10^{9} \mathrm{~cm}, 1.2 \times 10^{9} \mathrm{~cm}, 6 \times 10^{9} \mathrm{~cm}$ long, respectively. The $Z$ direction is perpendicular to the solar surface. The mesh is uniformly spaced along the three directions with $512 \times 128 \times 512$ cells, and a cell size of $\sim 80 \times 90 \times 120 \mathrm{~km}$, a good compromise between resolution in all directions (the domain is larger along $Z$ ) and computational times. The blobs are resolved well enough (their diameter is 30-40 cells) and the initial atmosphere has been checked to be steady with this resolution. The ambient atmosphere is a stratified corona linked to a much denser chromosphere through a steep transition region. The corona is a hydrostatic atmosphere (Rosner et al. 1978) that extends vertically for $10^{10} \mathrm{~cm}$. The chromosphere is hydrostatic and isothermal at $10^{4} \mathrm{~K}$ and its density is $\sim 10^{16} \mathrm{~cm}^{-3}$ at the bottom. The atmosphere is plane-parallel along $Z$. The coronal pressure ranges between $0.29 \mathrm{dyn}^{-2}$ at the top of the transition region and $0.12 \mathrm{dyn}^{-2}$ at $Z=10.5 \times 10^{9} \mathrm{~cm}$. The density and temperature are $\sim 2.2 \times 10^{8} \mathrm{~cm}^{-3}$ and $\sim 2 \times 10^{6} \mathrm{~K}$ at $Z=10.5 \times 10^{9} \mathrm{~cm}$ (Reale 2014). The falling blobs and the atmosphere are very similar to one of the models in Petralia et al. (2016; "dense model"), which are constrained from the observation and therefore realistic. Initially, the four blobs are at a height in the range $3.5<Z<4.5 \times 10^{9} \mathrm{~cm}$, and at a distance in a range $2.5<X<4 \times 10^{9} \mathrm{~cm}$ from the left boundary side, close to the upper right corner.

Figure 1 shows our initial conditions. For simplicity, we consider spherical blobs, with a radius between $1.4-2 \times 10^{8} \mathrm{~cm}$, typical of those in the eruption on 7 June 2011, temperature $T=10^{4} \mathrm{~K}$ and density $10^{10} \mathrm{~cm}^{-3}$, also typical of prominences (e.g. Labrosse et al. 2010; Parenti 2014). We assume that the blobs are optically thick. We expect that the timescales of the radiative transfer from the blobs (not included in the model) is much longer than that of the outside optically thinner plasma (included in the model), which in turn is much longer (e.g. Cargill et al. 1995; Reale 2014) than the very short timescale of the dynamics (only several minutes). Their initial speed is $v=300 \mathrm{~km} \mathrm{~s}^{-1}$. In one simulation, their motion is aligned cellby-cell with the magnetic field. The field intensity is $\sim 170 \mathrm{G}$ at the top of the transition region and $\sim 15 \mathrm{G}$ at the initial height of the blobs. In the other simulation, the magnetic field is weaker here, that is, $\sim 35 \mathrm{G}$ and $\sim 3 \mathrm{G}$, respectively, and the speed of the blobs is set uniform and not totally aligned with the field, but it lies in the $X Z$ plane: two have a horizontal initial direction, the other two have an inclination of $45^{\circ}$ downwards.

Boundary conditions are reflective at the left end of the $X$ axis, the magnetic field is forced to be perpendicular to the boundary at the right end. For the other quantities, we set a zero gradient. Fixed conditions were set at the lower end of the $Z$ axis

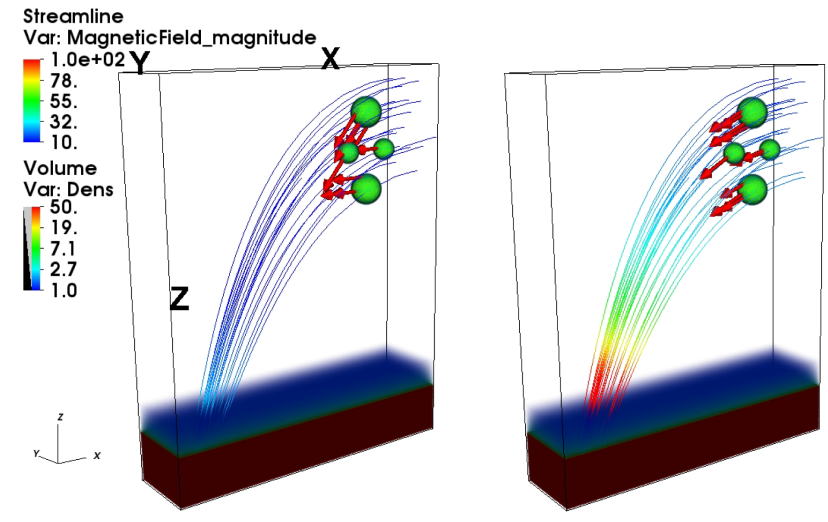

Fig. 1. Initial conditions of the two case simulations. Volume rendering of the density $\left(10^{9} \mathrm{~cm}^{-3}\right.$, logarithmic scale). Some magnetic field lines ( $\mathrm{G}$, coloured by field intensity) and blobs with initial velocity (red arrows) not aligned (left) and aligned (right) with the field lines are shown.

and a zero gradient at the upper end, except for the magnetic field that is fixed. The same conditions were set at the far end of the $Y$ axis. The computational domain was symmetric to a plane in $Y=0$, therefore we simulated half a domain and set reflective conditions at the lower end of the $Y$ axis.

We now describe the evolution of the flowing blobs, starting from those with an initial speed that is not aligned with the magnetic field, shown in Fig. 2 and the associated movie. This case is similar to those illustrated in Petralia et al. (2016), where the blobs crossed a closed magnetic field while falling. The propagation along the field lines is presented for comparison and shows a striking qualitative difference from the other one; this is the main motivation for this work. The initial speed of the blobs $\left(v=300 \mathrm{~km} \mathrm{~s}^{-1}\right)$ is not far from a typical freefall speed from large heights and larger than the local coronal sound speed $\left(c_{\mathrm{s}}=\sqrt{\gamma p / \rho} \sim 200 \mathrm{~km} \mathrm{~s}^{-1}\right)$, which means that shocks are generated immediately. These are slow-mode shocks that do not perturb the magnetic field and propagate along the magnetic field lines ahead of the blobs. In spite of the initial temperature jump at the transition region from the blob to the corona, the large difference in the heat capacity causes the conducted energy to be gradually radiated away, while the blobs dynamics dominate the entire evolution, as shown in previous work (Petralia et al. 2016). However, the blobs themselves do not move parallel to the magnetic field, perturbing and warping it strongly in a few seconds. The ram pressure carried by the blobs is $p_{\text {ram }}=\rho v^{2} \sim 20 \mathrm{dyn} \mathrm{cm}^{-2}$, much higher than the field pressure $B^{2} / 8 \pi \sim 0.3 \mathrm{dyn} \mathrm{cm}^{-2}$; the magnetic tension gives the field enough stiffness to channel the blobs. The net effect is that the moving blobs produce a tailspin that travels along the field lines. Measuring the distance and time taken to arrive at the chromosphere, the speed of this perturbation is $\sim 700 \mathrm{~km} \mathrm{~s}^{-1}$, meaning that it is an Alfvén wave that moves at an average speed $\left(v_{\mathrm{A}}=B / \sqrt{4 \pi \rho}\right)$ in a medium with density $7 \times 10^{8} \mathrm{~cm}^{-3}$ and magnetic field $\sim 10 \mathrm{G}$, reasonable average conditions for the medium where the perturbation is propagating. No MHD instabilities develop, the magnetic field is strong enough to suppress them (see Appendix A). While dragging the field lines, the misaligned and non-uniform motion of the blobs mixes them, and, as metal chords, they soon have a feedback on the blobs, mixing them in turn. As a result, the blobs rapidly lose their initial shape and even their single identity. They first form two separate conglomerates in the initial $30 \mathrm{~s}$, and the conglomerates travel along 

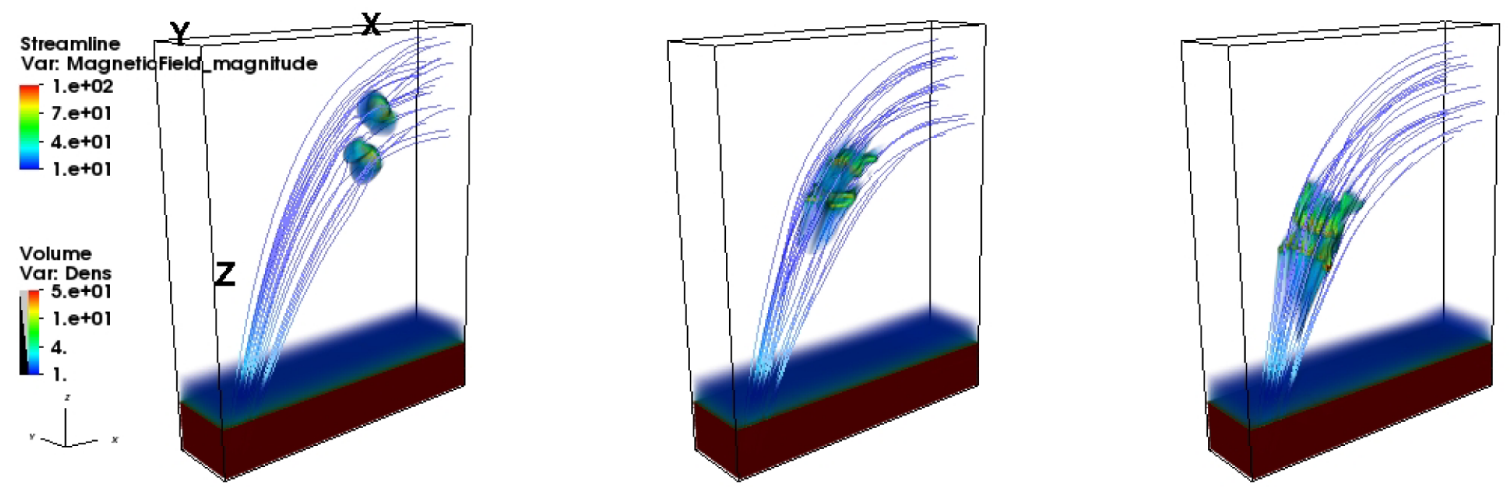

Fig. 2. Simulation of blobs not fully channelled by the magnetic field: volume rendering of the density at times $t=20,60$, and $100 \mathrm{~s}$ as in Fig. 1 . The temporal evolution (movie) is available online.
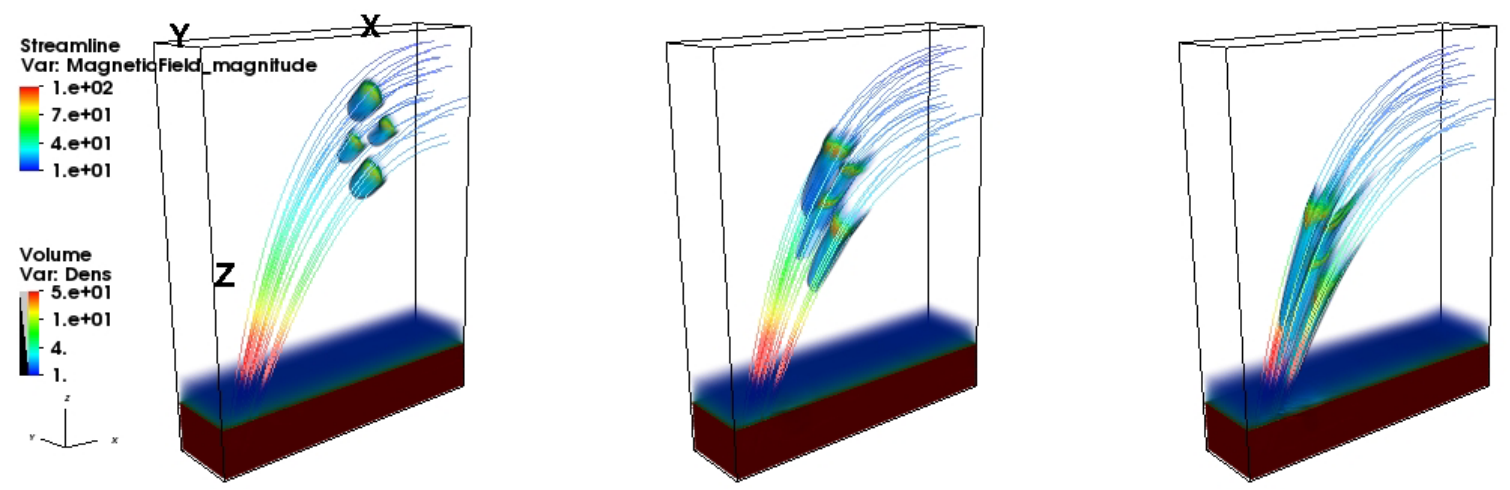

Fig. 3. Same as Fig. 2, but for the simulation of blobs that are fully channelled by the field and for the temporal evolution (movie).

the tube. They are progressively squashed and elongated into a waterfall-like shape, and in $\sim 2$ min they practically coalesce into a single blurred and filamented cloud, as shown in Fig. 2 and in the associated movie. In the meantime, they still flow along the magnetic tube toward the chromosphere. The return shock from the chromosphere contributes to further disrupt and mix the downflowing cloud. At the end of the shuffling, the identity of the blob is completely lost, what remains is a highly inhomogeneous flow structured into filaments that move chaotically along the field lines until they hit the surface in $200 \mathrm{~s}$.

We have checked that we obtain a similar evolution both for blobs with diverging velocities and for a single blob with an initial speed not aligned to the magnetic field lines, that is, the blobs are shuffled by the field and are disrupted.

Figure 3 and the associated movie show the propagation of blobs with a motion that initially is strictly aligned with the magnetic field lines. The velocity and the atmosphere conditions are equal to the previous case, so that the generation and propagation of the slow mode shocks are the same: once they are generated, they propagate along the magnetic field lines. In this case, the magnetic field intensity is five times greater than in the previous case, thus the magnetic field efficiently channels the blobs, and it is not perturbed significantly. The blobs simply flow along the magnetic field lines as the slow mode shocks do. No magnetic perturbation mixes the blobs, they remain compact during the motion. Their shape only varies because the magnetic channel changes its cross section and direction along the propagation. The blobs do not merge and therefore do not lose their identity during the propagation, as is clearly shown in Fig. 3.
Figure 4 emphasises the difference between the evolution of the misaligned and aligned motions. It shows cross sections of the density (Figs. 2 and 3) in vertical $Y Z$ planes. The images are taken at slightly different times, that is, $t=100 \mathrm{~s}$ and $90 \mathrm{~s}$, when the blobs are approximately in the same $Z$ range (the velocity component along the field lines is slightly different in the two cases). The figure very clearly shows that the evolution is different: a single but structured cloud versus three distant and separate blobs.

\section{Discussion and conclusions}

We studied the different propagations of fast plasma fragments flowing parallel to a coronal magnetic field from others flowing with a tilted direction through detailed 3D MHD modeling. Here we use the same model as in Petralia et al. (2016) to describe the propagation of dense and cold blobs of plasma moving in a magnetized solar atmosphere (including both the chromosphere and the corona). The model includes the effect of the gravity, optically thin radiative losses, thermal conduction along the field lines, and magnetic induction. The magneto-hydrodynamic equations were solved numerically (PLUTO code) in 3D Cartesian geometry. We compared two similar simulations of blobs flowing inside a magnetic field anchored in the solar surface. In one, their motion is fully channelled by the magnetic field, in the other it is only partially channelled because of the initial direction of the motion and because of the strength of the field. We changed the initial conditions of the blobs as little as possible from one case to the other. The 

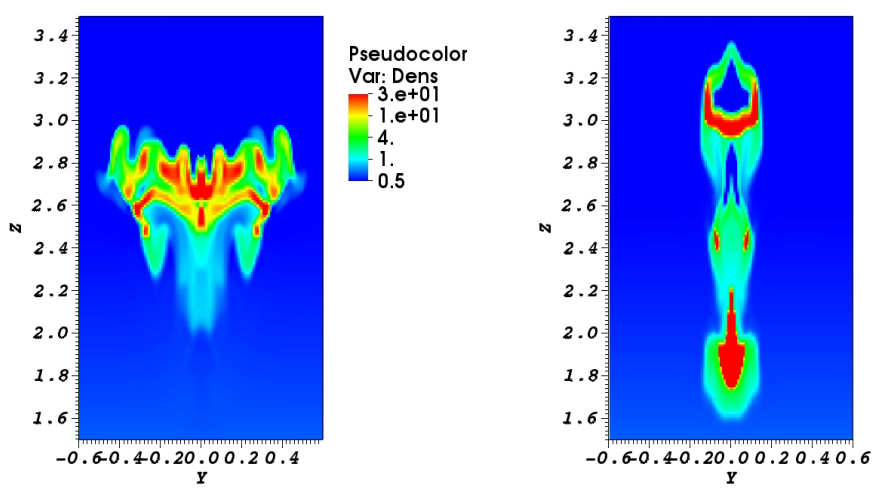

Fig. 4. Density $\left(10^{9} \mathrm{~cm}^{-3}\right.$, logarithmic scale $)$ in a plane $Y Z$ across the blobs for misaligned (left) at $t=100 \mathrm{~s}$ and $X=1.4 \times 10^{9} \mathrm{~cm}$, and for aligned blob motion (righ) at $t=90 \mathrm{~s}$ and $X=1.5 \times 10^{9} \mathrm{~cm}$.

compromise was to use the same initial velocity, which the blobs may acquire when they flow inside very large arches, such as in huge prominences. The evolution that we find is strikingly different. In the fully aligned case, the blobs and the slow-mode shocks flow along the field lines and do not perturb the intense magnetic field. The blobs remain compact and move inside independent magnetic channels. In the misaligned case, the shuffling of the field lines driven by the blobs creates a feedback on the blobs themselves and mixes them. At the same time, the conglomeration is structured into thinner filaments. In this case, it is impossible to establish the native channel or the initial shape of the blobs, they lose their identity completely. Misaligned propagation is also an efficient way to excite fast Alfvén wave fronts, which travel ahead of the cloud, in addition to shocks.

In summary, this work highlights the possible back-effect of the confining magnetic field on the propagation of fragmented flows inside it. When they are perfectly channelled, plasma fragments keep their identity as single blobs with no mixing, and the magnetic field is left unchanged as well. When there is some misalignment, the magnetic field can react with a shuffling of the field lines that mixes and merges the fragments, thus changing the plasma configuration completely. This represents a very effective mechanism of plasma mixing in the presence of a magnetic field, different from standard shear-like instabilities. The field lines can be effectively shuffled by irregular plasma motion and its feedback to the plasma is naturally chaotic. We may wonder which is the most common situation: aligned or misaligned fragment motion. We expect that if the plasma is confined since the beginning and the magnetic field does not change much along the track, the motion should be mostly aligned to the field and even more if blob velocity is lower, as in the coronal rain, in which similar blobs fall by gravity and reach loop footpoints with a velocity of about $60 \mathrm{~km} \mathrm{~s}^{-1}$ (Fang et al. 2013, 2015; Moschou et al. 2015). All our evolution occurs on timescales about two orders of magnitudes shorter than in these other studies, so that our results might only apply to the very final stages of their modelling. On the other hand, downfalling from large distances through a significantly changing magnetic field might result in misaligned fragment motion. A situation like this may occur in the accretion onto young protostars from circumstellar disks, both at the flow origin (disk) and close to the flow impact, where the magnetic field of the star might become very complex. This process might therefore lead to further mixing of downflows and increase their fine substructure. For this exploratory work, the initial conditions of our simulations differ in both the speed alignment and in the strength of the ambient magnetic field. Our aim here is to show that the moving blobs can have two different destinies, but we did not explore the conditions necessary to switch between the two in detail. This exploration is postponed to more extended work. Although this work mainly addresses downfall motions, it might be more general, and may in particular also involve the case of upflows. This is to be addressed in future work.

Acknowledgements. A.P., F.R., and S.O. acknowledge support from the Italian Ministero dell'Università e Ricerca. PLUTO is developed at the Turin Astronomical Observatory in collaboration with the Department of Physics of the Turin University. We acknowledge the HPC facilities SCAN, of the INAF-Osservatorio Astronomico di Palermo, the CINECA Award HP10B59JKR for the availability of high-performance computing resources and support.

\section{References}

Antolin, P., \& Rouppe van der Voort, L. 2012, ApJ, 745, 152

Bertout, C., Basri, G., \& Bouvier, J. 1988, ApJ, 330, 350

Cargill, P. J., Mariska, J. T., \& Antiochos, S. K. 1995, ApJ, 439, 1034

Chen, P. F. 2011, Liv. Rev. Sol. Phys., 8, 1

Colombo, S., Orlando, S., Peres, G., Argiroffi, C., \& Reale, F. 2016, A\&A, 594, A93

Dere, K. P. 2009, A\&A, 497, 287

Fang, X., Xia, C., \& Keppens, R. 2013, ApJ, 771, L29

Fang, X., Xia, C., Keppens, R., \& Van Doorsselaere, T. 2015, ApJ, 807, 142

Field, G. B. 1965, ApJ, 142, 531

Innes, D. E., Cameron, R. H., Fletcher, L., Inhester, B., \& Solanki, S. K. 2012, A\&A, 540, L10

Kleint, L., Antolin, P., Tian, H., et al. 2014, ApJ, 789, L42

Labrosse, N., Heinzel, P., Vial, J.-C., et al. 2010, Space Sci. Rev., 151, 243

Landi, E., Del Zanna, G., Young, P. R., Dere, K. P., \& Mason, H. E. 2012, ApJ, 744, 99

Matsakos, T., Chièze, J.-P., Stehlé, C., et al. 2013, A\&A, 557, A69

Mignone, A., Bodo, G., Massaglia, S., et al. 2007, ApJS, 170, 228

Mignone, A., Zanni, C., Tzeferacos, P., et al. 2012, ApJS, 198, 7

Moschou, S. P., Keppens, R., Xia, C., \& Fang, X. 2015, Adv. Space Res., 56, 2738

Orlando, S., Bonito, R., Argiroffi, C., et al. 2013, A\&A, 559, A127

Parenti, S. 2014, Liv. Rev. Sol. Phys., 11, 1

Parker, E. N. 1953, ApJ, 117, 431

Petralia, A., Reale, F., Orlando, S., \& Testa, P. 2016, ApJ, 832, 2

Priest, E. 2014, in Magnetohydrodynamics of the Sun (Cambridge, UK: Cambridge University Press)

Reale, F. 2014, Liv. Rev. Sol. Phys., 11, 4

Reale, F., Orlando, S., Testa, P., et al. 2013, Science, 341, 251

Reale, F., Orlando, S., Testa, P., Landi, E., \& Schrijver, C. J. 2014, ApJ, 797, L5

Rosner, R., Tucker, W. H., \& Vaiana, G. S. 1978, ApJ, 220, 643

Uchida, Y., \& Shibata, K. 1984, PASJ, 36, 105

van Driel-Gesztelyi, L., Baker, D., Török, T., et al. 2014, ApJ, 788, 85

Webb, D. F., \& Howard, T. A. 2012, Liv. Rev. Sol. Phys., 9, 3 


\section{Appendix A: MHD shear instabilities}

\section{A.1. Kelvin-Helmoltz instability}

When a heavier fluid (blobs) in motion is sustained against a lighter fluid (the corona) by the magnetic field, Kelvin-Helmoltz instabilities can arise. They are suppressed when the magnetic field is strong enough to satisfy the condition (Priest 2014, and references therein)

$\frac{B_{-}^{2}+B_{+}^{2}}{4 \pi \rho_{-} \rho_{+}}\left(\rho_{-}+\rho_{+}\right) \geq\left(U_{-}-U_{+}\right)^{2}$,

where subscripts - and + denote the variables inside and outside the blobs, respectively, $B$ is the magnetic field, $\rho$ is the mass density, and $U$ is the velocity.

Considering that the blob density $\left(n_{\mathrm{b}} \approx 10^{10} \mathrm{~cm}^{-3}\right)$ is much higher than the coronal ambient density $\left(n_{\mathrm{c}} \approx 3 \times 10^{8} \mathrm{~cm}^{-3}\right)$, the magnetic field intensity is $\sim 3 \mathrm{G}$, for the misaligned blobs, and does not change much at the interface of blob and corona, the ambient medium is static, Eq. (A.1) can be simplified to

$\frac{2 B^{2}}{4 \pi n_{\mathrm{b}} \mu m_{\mathrm{H}}} \geq U^{2}$, where $\mu m_{\mathrm{H}}$ is the mean atomic mass. We obtain $3 \times 10^{15}>10^{15}$ and an even larger difference for the aligned blobs where the magnetic field is 25 times more intense. In our simulations Kelvin-Helmoltz instabilities are therefore efficiently suppressed by the magnetic field.

\section{A.2. Rayleigh-Taylor instability}

The high ratio between the density of the blobs and the ambient corona could make the separation of the blob-to-corona layer subject to the Rayleigh-Taylor instability. The wave vectorof such perturbations is smaller than a critical threshold given by (Priest 2014, and references therein)

$k<k_{\mathrm{c}}=\frac{4 \pi\left(\rho_{+}-\rho_{-}\right) g_{\odot}}{2 B^{2}}$,

with subscripts as in Eq. (A.1). This critical value leads to a lower limit for the characteristic length of the perturbation, which we estimated to be $L_{\mathrm{c}}=2 \pi / k_{\mathrm{c}}>10^{11} \mathrm{~cm}$. This is much larger than the size of the blobs. 